(C) 2001 International Press

Adv. Theor. Math. Phys. 5 (2001) 923-946

\title{
The Minimal Unitary Representation of $E_{8(8)}{ }^{\dagger}$
}

M. Günaydin ${ }^{a \ddagger}$, K. Koepsell ${ }^{b}$, H. Nicolai ${ }^{b}$

\author{
${ }^{a}$ Penn State University, \\ Physics Department, \\ University Park, PA 16802, USA \\ murat@phys.psu.edu \\ ${ }^{b}$ Max-Planck-Institut für Gravitationsphysik, \\ Albert-Einstein-Institut, \\ Mühlenberg 1, D-14476 Golm, Germany \\ koepsell@aei.mpg.de, nicolai@aei.mpg.de
}

\begin{abstract}
We give a new construction of the minimal unitary representation of the exceptional group $E_{8(8)}$ on a Hilbert space of complex functions in 29 variables. Due to their manifest covariance with respect to the $E_{7(7)}$ subgroup of $E_{8(8)}$ our formulas are simpler than previous realizations, and thus well suited for applications in superstring and $\mathrm{M}$ theory.
\end{abstract}

e-print archive: http://xxx.lanl.gov/hep-th/0109005

${ }^{\dagger}$ This work was supported in part by the NATO collaborative research grant CRG. 960188.

${ }^{\ddagger}$ Work supported in part by the National Science Foundation under grant number PHY-0099548. 


\section{Introduction}

In this paper, we present a new construction of the minimal unitary representation of $E_{8(8)}$ (i.e. the maximally split real form of the exceptional Lie group $E_{8}$ ) on a Hilbert space of complex functions in 29 variables $^{1}$. The minimal realizations of classical Lie algebras and of $G_{2}$ were given by Joseph a long time ago $[22,23]$. The existence of the minimal unitary representation of $E_{8(8)}$ was first proved by Vogan [35] who located it within the framework of Langland's classification. Later, the minimal unitary representations of all simply laced groups, including $E_{8(8)}, E_{7(7)}$ and $E_{6(6)}$ were constructed by Kazhdan and Savin [25], and Brylinski and Kostant $[5,6,7,8]$ by rather different methods. Gross and Wallach gave yet another construction of the minimal representation of $E_{8(8)}$ as well as for all exceptional groups of real rank four [17]. For the exceptional group $E_{8(8)}$ the minimal orbit is 58 dimensional, corresponding to its quotient by a distinguished parabolic subgroup. The representations of $E_{8(8)}$ over the spaces of functions on the 128 dimensional coset space $E_{8(8)} / S O(16)$ were studied in [1].

While formulas for $G_{2}$ similar to the ones derived here for $E_{8(8)}$ can be found in [6], however, an explicit realization of the simple root (Chevalley) generators in terms of pseudo-differential operators for the simply laced exceptional groups was given only very recently [26], together with the spherical vectors necessary for the construction of modular forms. We present here an alternative realization of $E_{8(8)}$, which has the advantage of yielding very compact formulas, in contradistinction to the rather complicated expressions obtained by multiple commutation of the simple root generators [27]. The main reason for the relative simplicity of our final expressions (7) and (12) is their manifest $E_{7(7)}$ covariance, in spite of the fact that $E_{7(7)}$ is not realized linearly. The compactness of our formulas makes them especially suitable for applications in string and $M$ theory which have primarily motivated the present work.

Although the present article is mainly addressed to a physicists' audience, let us first summarize the main results in a somewhat more mathematical language. The minimal representation of a noncompact group $G$ corresponds, in general, to the quantization of its smallest co-adjoint orbit. In the case of $E_{8(8)}$ the minimal co-adjoint orbit is 58-dimensional. Starting from the 5-graded decomposition of the associated Lie algebra $\mathfrak{e}_{8}$ given in eq. (4) below, it can be obtained simply as the co-adjoint orbit of the highest root generator (designated by $E$ in eq. (6)). In this 5 -graded decomposition

\footnotetext{
${ }^{1} \mathrm{~A}$ preliminary account of some of the results presented in section 2 has already appeared in [29].
} 
of $\mathfrak{e}_{8}$ one can readily identify a maximal non-semisimple subalgebra $\mathfrak{p}$ as the annihilator of this generator, i.e.

$$
[\mathfrak{p}, E]=0
$$

The subalgebra $\mathfrak{p}$ is generated by the grade zero subalgebra $\mathfrak{e}_{7}$ together with the grade 1 and grade 2 elements of the full $\mathfrak{e}_{8}$ Lie algebra (i.e. the generators $\left(E_{i j}, E^{i j}\right)$ and $E$ in eq. (6)). The orthogonal complement of this parabolic subalgebra is a 58 dimensional nilpotent subalgebra $\mathfrak{n}$. In the notation of section 2, the latter is generated by the grade 0 element $H$, the grade $(-1)$ elements $\left(F_{i j}, F^{i j}\right)$, and the grade $(-2)$ element $F$. Acting with this nilpotent subalgebra on the generator $E$ corresponding to the highest root one obtains the generators corresponding to the minimal orbit. Thus we see that the minimal orbit can be identified with the coset space $E_{8(8)} / \mathfrak{P}$ where $\mathfrak{P}$ is the parabolic subgroup generated by $\mathfrak{p}$.

We were led to this minimal unitary representation rather naturally from the novel geometric realization of $E_{8(8)}$ on $\mathbb{R}^{57}$ found in our previous work [18]. As shown there, the exceptional groups can be realized via conformal or quasiconformal transformations leaving invariant generalized "light-cones"; these transformations are analogous to the non-linear action of the Möbius group on the real line. Our construction relied essentially on the connection with Jordan algebras and Freudenthal triple systems. To proceed from there to the unitary realization on a suitable (infinite dimensional) Hilbert space of functions, we must first identify a phase space realization of this system and then quantize it. For the $E_{7(7)}$ subgroup this phase space realization involves 28 coordinates $X^{i j}$ and 28 momenta $P_{i j}$, such that all $E_{7(7)}$ variations can be realized via the canonical action

$$
\delta_{\mathcal{Q}} X \equiv\{\mathcal{Q}, X\}_{\text {P.B. }}, \quad \delta_{\mathcal{Q}} P \equiv\{\mathcal{Q}, P\}_{\text {P.B. }}
$$

where $\mathcal{Q}=\mathcal{Q}(X, P)$ is the appropriate $E_{7(7)}$ charge. For the canonical realization of the full $E_{8(8)}$ we have to add the momentum $p$ conjugate to the 57th coordinate $y$, and replace the Möbius action on $y$ by the symplectic realization acting on the two dimensional phase space spanned by $y$ and $p$. The minimal unitary representation of $E_{8(8)}$ is then obtained by quantization, i.e. replacement of the classical momenta by differential operators. This requires in addition a prescription how to deal with the ordering ambiguities arising in the non-linear expressions for the Lie algebra generators. The latter are then realized as self-adjoint operators on some dense subspace of $\mathrm{L}^{2}\left(\mathbb{R}^{29}\right)$. This coordinate space (Schrödinger) representation is reformulated in section 3 as an oscillator realization in terms of annihilation and creation operators acting in a particle basis. By going to the corresponding 
coherent state basis of this oscillator realization labelled by 29 complex coordinates one obtains the Fock-Bargmann realization of the minimal unitary representation over an Hilbert space of holomorphic functions.

The irreducibility of the minimal representation is put in evidence by showing that the quadratic Casimir operator of $E_{8(8)}$, when expressed in terms of either the coordinate or the oscillator basis reduces to a $c$-number; a general argument shows that likewise the higher order Casimirs reduce to $c$ numbers in our realization. When restricted to the subgroup $E_{7(7)} \times S L(2, \mathbb{R})$ the minimal representation decomposes into an infinite sum (actually an integral, see [5]) of irreducible representations of $E_{7(7)} \times S L(2, \mathbb{R})$. Remarkably, the quadratic Casimir of $E_{7(7)}$ obtained from the minimal realization is identical with the quartic invariant of $E_{7(7)}$ when expressed as a function of the 28 coordinate and 28 momentum operators (or equivalently, the 28 annihilation and 28 creation operators in the oscillator basis). The irreducible representations of $S L(2, \mathbb{R})$ that occur in the decomposition of the minimal representation $E_{8(8)}$ w.r.t. $E_{7(7)} \times S L(2, \mathbb{R})$ are all labelled by the eigenvalues of this quartic invariant.

As already mentioned our chief aim with the present work is to present the results in such a way that they can be readily applied in the context of string and $M$ theory (for instance, readers familiar with [10] should have no difficulties following our exposition). In particular, our results apply in the context of the (super)conformal quantum mechanics description of quantum black holes $[11,9,30]$. In that work, unitary representations of $S L(2, \mathbb{R})$ played a crucial role in the classification of physical states. It is therefore an obvious idea to extend these concepts to maximal supergravity. Indeed, the candidate Hamiltonian which we obtain as one of the $E_{8(8)}$ Lie algebra generators reads

$$
L_{0}=\frac{1}{4}\left[p^{2}+y^{2}+4 y^{-2} I_{4}(X, P)\right]
$$

which is precisely of the form studied by the authors of $[11,9,30]$, with the only difference that they have a (coupling) constant instead of the differential operator $I_{4}(X, P)$. Here $I_{4}$ is the quartic invariant of $E_{7(7)}$ expressed as a function of the $28+28$ variables $X^{i j}$ and $P_{i j}$; when acting on one of the irreducible subrepresentations of $E_{7(7)}$ under its subgroup $E_{7(7)} \times S L(2, \mathbb{R})$ it yields the associated eigenvalue of the quadratic $E_{7(7)}$ Casimir. As shown for instance in [18] in the context of the classical theory, the quartic invariant can assume both positive and negative values, and vanishes for $\frac{1}{2}$ or $\frac{1}{4}$ BPS black hole solutions of $N=8$ supergravity [24, 14, 13]. It is therefore tempting to interpret (3) as the effective Hamiltonian describing (in some approximation) $N=8$ quantum black holes, such that every subrepresentation with a fixed eigenvalue of the operator $I_{4}$ is identified with the 
space of physical states associated with the corresponding black hole solution of $N=8$ supergravity. An interpretation along similar lines has also been suggested in [16]. Interestingly, for the vanishing eigenvalues of $I_{4}$, the Hamiltonian simplifies drastically, and the state space reduces to the well known singleton representation of $S L(2, \mathbb{R})$.

Another (and possibly related) physical application of minimal representations has been outlined in [26]. That work evolved from an earlier attempt to determine the $R^{4}$ corrections directly from the supermembrane and to understand them in terms of so-called "theta-correspondences" [31]; see also [34] for a related attempt to come to grips with the non-linearities of the supermembrane.

\section{Coordinate Space (Schrödinger) Representation}

We first recall some basic features of the non-linear realization of $E_{8(8)}$ on $\mathbb{R}^{57}$ coordinatized by 57 real variables $\left\{X^{i j}, X_{i j}, y\right\}(i, j=1, \ldots, 8)$, see [18] for our notations and conventions, and further details. A key ingredient in that construction was the 5-graded decomposition of $E_{8(8)}$ w.r.t. its subgroup $E_{7(7)} \times S L(2, \mathbb{R})$. Denoting its Lie algebra by $\mathfrak{e}_{8}$ we have

$$
\mathfrak{e}_{8}=\mathfrak{g}^{-2} \oplus \mathfrak{g}^{-1} \oplus \mathfrak{g}^{0} \oplus \mathfrak{g}^{+1} \oplus \mathfrak{g}^{+2} .
$$

An important property of this decomposition is the fact that the subspaces of grade -1 and -2 together form a maximal Heisenberg subalgebra. The corresponding generators $E^{i j}, E_{i j} \in \mathfrak{g}^{-1}$ and $E \in \mathfrak{g}^{-2}$ obey the commutation relations

$$
\left[E^{i j}, E_{k l}\right]=2 \mathrm{i} \delta_{k l}^{i j} E .
$$

Obviously, this algebra can be realized as a classical Poisson algebra on a phase space with 28 coordinates $X^{i j}$ and 28 momenta $P_{i j} \equiv X_{i j}$, and one extra real coordinate $y$ to represent the central term. We have

$$
E^{i j}:=y X^{i j}, \quad E_{i j}:=y P_{i j}, \quad E:=\frac{1}{2} y^{2} .
$$

It is then straightforward to determine the generators of the $\mathfrak{e}_{7}$ subalgebra in terms of these phase space variables (for instance by requiring that they reproduce the $E_{7(7)}$ variations via (2)). They are realized by the following $63+70$ bilinear expressions in $X^{i j}$ and $P_{i j}$

$$
\begin{aligned}
G_{j}^{i} & :=2 X^{i k} P_{k j}+\frac{1}{4} X^{k l} P_{k l} \delta_{j}^{i}, \\
G^{i j k l} & :=-\frac{1}{2} X^{[i j} X^{k l]}+\frac{1}{48} \epsilon^{i j k l m n p q} P_{m n} P_{p q} .
\end{aligned}
$$


To extend this canonical realization to the full $E_{8(8)}$, we need one more variable, the momentum $p$ conjugate to $y$. Combining the symplectic realization of $S L(2, \mathbb{R})$ with the non-linear variations (23) and (24) of [18] we can then deduce the classical (phase space) analogs of the $E_{8(8)}$ generators. Owing to the non-linearity of the realization, the resulting expressions are not only quadratic, but go up to fourth order in the phase space variables $X^{i j}$ and $P_{i j}$, and moreover contain inverse powers of $y$. Thus all generators of $E_{8(8)}$ can be realized on a 58-dimensional phase space coordinatized by $\left\{X^{i j}, P_{i j} ; y, p\right\}$. The minimal unitary 29 dimensional representation of $E_{8(8)}$ is then obtained by quantization, i.e. by introducing the usual momentum operators obeying the canonical commutation relations

$$
\left[X^{i j}, P_{k l}\right]=\mathrm{i} \delta_{k l}^{i j}, \quad[y, p]=\mathrm{i} .
$$

In elevating the $E_{8(8)}$ generators to quantum operators the only problem $v i s$ - $\grave{a}$-vis the classical phase space description is the non-commutativity of the coordinate and momentum operators, which requires some ordering prescription (we note, that the $E_{7(7)}$ generators (7) are insensitive to re-ordering the coordinates and momenta). Since we are interested in finding a unitary representation we insist that all operators are hermitean w.r.t. to the standard scalar product on $\mathrm{L}^{2}\left(\mathbb{R}^{29}\right)$. We thus arrive at a unitary representation of the $E_{8(8)}$ Lie algebra in terms of self-adjoint operators acting on (some dense subspace of) a Hilbert space of complex functions in 29 real variables $\left\{X^{i j}, y\right\}$. We emphasize that this realization requires complex functions, for the same reason that the Schrödinger representation of the one-dimensional point particle requires complex wave functions ${ }^{2}$.

Before writing out the $E_{8(8)}$ generators, let us list the $E_{7(7)}$ commutation relations

$$
\begin{aligned}
{\left[G^{i}{ }_{j}, G^{k}{ }_{l}\right] } & =\mathrm{i} \delta_{j}^{k} G_{l}^{i}-\mathrm{i} \delta_{l}^{i} G_{j}^{k}, \\
{\left[G^{i}{ }_{j}, G^{k l m n}\right] } & =-4 \mathrm{i} \delta_{j}^{[k} G^{l m n] i}-\frac{\mathrm{i}}{2} \delta_{j}^{i} G^{k l m n}, \\
{\left[G^{i j k l}, G^{m n p q}\right] } & =\frac{\mathrm{i}}{36} \epsilon^{i j k l s[m n p} G^{q]}{ }_{s} .
\end{aligned}
$$

Observe that only the $S L(8, \mathbb{R})$ subgroup acts by linear transformations; its maximal compact subgroup $S O(8)$ is generated by $G_{i j}^{-}$, where

$$
G_{i j}^{ \pm}:=\frac{1}{2}\left(G^{i}{ }_{j} \pm G^{j}{ }_{i}\right)
$$

\footnotetext{
${ }^{2}$ As we show in the next section the generators of $E_{8(8)}$ can be rewritten in terms of bosonic annihilation and creation operators. In the corresponding coherent state basis the Hilbert space will involve holomorphic functions in 29 variables.
} 
For later convenience, we also define

$$
G_{i j k l}:=\frac{1}{24} \epsilon_{i j k l m n p q} G^{m n p q},
$$

and the selfdual and anti-selfdual combinations

$$
G_{i j k l}^{ \pm}:=\frac{1}{2}\left(G^{i j k l} \pm G_{i j k l}\right)
$$

where the $G_{i j k l}^{-}$are compact and the $G_{i j k l}^{+}$non-compact.

The following generators extend this representation to the full $E_{8(8)}$ (we use the same notation for the elements of $\mathfrak{e}_{8}$ as in [18], in accordance with its 5-graded structure):

$$
\begin{aligned}
E:= & \frac{1}{2} y^{2} \\
E^{i j}:= & y X^{i j} \\
E_{i j}:= & y P_{i j}, \\
H:= & \frac{1}{2}(y p+p y), \\
F^{i j}:= & -p X^{i j}+2 \mathrm{i} y^{-1}\left[X^{i j}, I_{4}(X, P)\right] \\
= & -4 y^{-1} X^{i k} P_{k l} X^{l j}-\frac{1}{2} y^{-1}\left(X^{i j} P_{k l} X^{k l}+X^{k l} P_{k l} X^{i j}\right) \\
& +\frac{1}{12} y^{-1} \epsilon^{i j k l m n p q} P_{k l} P_{m n} P_{p q}-p X^{i j}, \\
F_{i j}:= & -p P_{i j}+2 \mathrm{i} y^{-1}\left[P_{i j}, I_{4}(X, P)\right] \\
= & 4 y^{-1} P_{i k} X^{k l} P_{l j}+\frac{1}{2} y^{-1}\left(P_{i j} X^{k l} P_{k l}+P_{k l} X^{k l} P_{i j}\right) \\
& -\frac{1}{12} y^{-1} \epsilon_{i j k l m n p q} X^{k l} X^{m n} X^{p q}-p P_{i j}, \\
F= & \frac{1}{2} p^{2}+2 y^{-2} I_{4}(X, P) .
\end{aligned}
$$

The hermiticity of all generators is manifest. Here $I_{4}(X, P)$ is the fourth order differential operator

$$
\begin{aligned}
I_{4}(X, P):= & -\frac{1}{2}\left(X^{i j} P_{j k} X^{k l} P_{l i}+P_{i j} X^{j k} P_{k l} X^{l i}\right) \\
& +\frac{1}{8}\left(X^{i j} P_{i j} X^{k l} P_{k l}+P_{i j} X^{i j} P_{k l} X^{k l}\right) \\
& -\frac{1}{96} \epsilon^{i j k l m n p q} P_{i j} P_{k l} P_{m n} P_{p q} \\
& -\frac{1}{96} \epsilon_{i j k l m n p q} X^{i j} X^{k l} X^{m n} X^{p q}+\frac{547}{16} .
\end{aligned}
$$

As a function of $X^{i j}$ and $P_{i j}$ this operator represents the quartic invariant of $E_{7(7)}$ because

$$
\left[G^{i}{ }_{j}, I_{4}(X, P)\right]=\left[G^{i j k l}, I_{4}(X, P)\right]=0 .
$$


We emphasize the importance of the ordering adopted in (13) for the vanishing of (the second of) these commutators. Orderings that differ from (13) by a term containing the Euler operator $i X^{i j} P_{i j}$ break $E_{7(7)}$ invariance. On the other hand, (14) is insensitive to re-orderings which differ from (13) only by a $c$-number, and in the absence of a preferred ordering there is thus no absolute significance to the additive constant appearing in the definition of $I_{4}$. For instance, an admissible re-ordering is

$$
-\frac{1}{6} G^{i}{ }_{j} G_{i}^{j}-G^{i j k l} G_{i j k l}=I_{4}(X, P)-\frac{323}{16} .
$$

This relation confirms our previous assertion that, when acting on a given representation of $E_{7(7)}, I_{4}$ is just the quadratic $E_{7(7)}$ Casimir invariant, up to an additive constant.

For the derivation of (12), we notc that, as already pointed out in [5], the crucial step is the determination of the operators $E$ and $F$ corresponding to the lowest and highest root of $E_{8(8)}$, respectively. The expressions for $F^{i j}$ and $F_{i j}$ then follow by commutation with $E^{i j}$ and $E_{i j}$. While the derivation of [5] relied on a generalization of the so-called "Capelli-identity", the form of our operators $E$ and $F$ follows directly from $E_{7(7)}$ invariance and a scaling argument, up to the additive constant in (13). The latter originates from the re-ordering required to bring the commutator of $F_{i j}$ and $F^{i j}$ into the "standard form" defined by the r.h.s. of (13).

As anticipated, all generators transform covariantly under the full $E_{7(7)}$ group.

$$
\begin{aligned}
& {\left[G^{i}{ }_{j}, E^{k l}\right]=\mathrm{i} \delta_{j}^{k} E^{i l}-\mathrm{i} \delta_{j}^{l} E^{i k}-\frac{\mathrm{i}}{4} \delta_{j}^{i} E^{k l},} \\
& {\left[G^{i}{ }_{j}, E_{k l}\right]=\mathrm{i} \delta_{k}^{i} E_{l j}-\mathrm{i} \delta_{l}^{i} E_{k j}+\frac{\mathrm{i}}{4} \delta_{j}^{i} E_{k l},} \\
& {\left[G^{i}{ }_{j}, F^{k l}\right]=\mathrm{i} \delta_{j}^{k} F^{i l}-\mathrm{i} \delta_{j}^{l} F^{i k}-\frac{\mathrm{i}}{4} \delta_{j}^{i} F^{k l},} \\
& {\left[G^{i}{ }_{j}, F_{k l}\right]=\mathrm{i} \delta_{k}^{i} F_{l j}-\mathrm{i} \delta_{l}^{i} F_{k j}+\frac{\mathrm{i}}{4} \delta_{j}^{i} F_{k l} .}
\end{aligned}
$$

The remaining part of $E_{7(7)}$ acts as

$$
\begin{aligned}
{\left[G^{i j k l}, E_{m n}\right] } & =-\mathrm{i} \delta_{m n}^{[i j} E^{k l]}, \\
{\left[G^{i j k l}, E^{m n}\right] } & =-\frac{i}{24} \epsilon^{i j k l m n p q} E_{p q}, \\
{\left[G^{i j k l}, F_{m n}\right] } & =-\mathrm{i} \delta_{m n}^{[i j} F^{k l]}, \\
{\left[G^{i j k l}, F^{m n}\right] } & =-\frac{i}{24} \epsilon^{i j k l m n p q} F_{p q} .
\end{aligned}
$$


The grading of the generators is given by the dilatation generator $H$

$$
\begin{aligned}
{[H, E] } & =-2 \mathrm{i} E, & {[H, F] } & =2 \mathrm{i} F, \\
{\left[H, E^{i j}\right] } & =-\mathrm{i} E^{i j}, & {\left[H, F^{i j}\right] } & =\mathrm{i} F^{i j}, \\
{\left[H, E_{i j}\right] } & =-\mathrm{i} E_{i j}, & {\left[H, F_{i j}\right] } & =\mathrm{i} F_{i j} .
\end{aligned}
$$

The remaining non-vanishing commutation relations are

$$
\begin{aligned}
& \left.\left[E^{i j}, F^{k l}\right]=12 \mathrm{i} G^{i j k l}, \quad\left[E^{i j}, F_{k l}\right]=4 \mathrm{i} \delta_{[k}^{[i} G^{j]} l\right]-\mathrm{i} \delta_{k l}^{i j} H, \\
& \left.\left[E_{i j}, F_{k l}\right]=-12 \mathrm{i} G_{i j k l}, \quad\left[E_{i j}, F^{k l}\right]=4 \mathrm{i} \delta_{[i}^{[k} G^{l]} j\right]+\mathrm{i} \delta_{k l}^{i j} H, \\
& {\left[E^{i j}, E_{k l}\right]=2 \mathrm{i} \delta_{k l}^{i j} E, \quad\left[F^{i j}, F_{k l}\right]=2 \mathrm{i} \delta_{k l}^{i j} F,} \\
& {\left[E, F^{i j}\right]=-\mathrm{i} E^{i j}, \quad\left[F, E^{i j}\right]=\mathrm{i} F^{i j},} \\
& {\left[E, F_{i j}\right]=-\mathrm{i} E_{i j}, \quad\left[F, E_{i j}\right]=\mathrm{i} F_{i j},} \\
& {[E, F]=\text { i } H \text {. }}
\end{aligned}
$$

The $E_{8(8)}$ commutation relations are the same as in [18], except that the structure constants carry an extra factor of i. As can be verified by computation of the Cartan Killing form from the structure constants that can be extracted from the above commutation relations, the maximal compact subgroup $S O(16)$ is generated by the following linear combinations of $E_{8(8)}$ generators

$$
G_{i j}^{-}, G_{i j k l}^{-}, E_{i j}+F^{i j}, E^{i j}-F_{i j}, E+F .
$$

This confirms that we are indeed dealing the split real form $E_{8(8)}$.

The quadratic $E_{8(8)}$ Casimir operator (in a convenient normalization) is a sum of three terms

$$
\mathcal{C}_{2}\left[E_{8(8)}\right]=\mathcal{C}_{2}[S L(2, \mathbb{R})]+\mathcal{C}_{2}\left[E_{7(7)}\right]+\mathcal{C}_{2}^{\prime}
$$

with

$$
\begin{aligned}
\mathcal{C}_{2}[S L(2, \mathbb{R})] & :=\frac{1}{2}\left(E F+F E-\frac{1}{2} H^{2}\right), \\
\mathcal{C}_{2}\left[E_{7(7)}\right] & :=-\frac{1}{2} G_{j}^{i} G^{j}{ }_{i}-3 G^{i j k l} G_{i j k l}, \\
\mathcal{C}_{2}^{\prime} & =\frac{1}{4}\left(E^{i j} F_{i j}+F_{i j} E^{i j}-E_{i j} F^{i j}-F^{i j} E_{i j}\right) .
\end{aligned}
$$

Here the terms in the first and second line represent the Casimir operators of the $S L(2, \mathbb{R})$ and $E_{7(7)}$ subalgebras, respectively. When substituting the explicit expressions in terms of coordinates and momenta for the generators, we obtain

$$
\begin{aligned}
\mathcal{C}_{2}[S L(2, \mathbb{R})] & =I_{4}-\frac{3}{16} \\
\mathcal{C}_{2}\left[E_{7(7)}\right] & =3 I_{4}-\frac{969}{16} \\
\mathcal{C}_{2}^{\prime} & =-4 I_{4}-\frac{237}{4}
\end{aligned}
$$


Hence all terms containing the operators $X^{i j}, P_{i j}, y$ and $p$ actually cancel, leaving us with a constant value for the minimal representation

$$
\mathcal{C}_{2}\left[E_{8(8)}\right]=-120
$$

which is the $E_{8(8)}$ analog of the result

$$
\mathcal{C}_{2}[S L(2, \mathbb{R})]=\frac{1}{4} g-\frac{3}{16}
$$

familiar from conformal quantum mechanics - except that there is no coupling constant any more for $E_{8(8)}$ that we can tune! Of course, unlike for the group $S L(2, \mathbb{R})^{3}$, this result does not by itself imply the irreducibility of the minimal representation of $E_{8(8)}$. To show that, we have to compute in addition the eigenvalues of the higher order $E_{8(8)}$ Casimir invariants. However, it is almost self-evident that these, too, will collapse to $c$-numbers when the coordinates and momenta are substituted, for the simple reason that we cannot build $E_{8(8)}$ invariants from the coordinate and momentum operators alone. This is in stark contrast to the singleton representation of $E_{7(7)}$ for which the coordinates and momenta do form a non-trivial (linear) representation of $E_{7(7)}$, permitting the construction of non-vanishing higher order $E_{7(7)}$ invariants. This provides an independent argument that the minimal representation of $E_{8(8)}$ is indeed irreducible.

We conclude this section by giving the Chevalley generators corresponding to the eight simple roots of $E_{8(8)}$, with the labeling indicated in the figure.

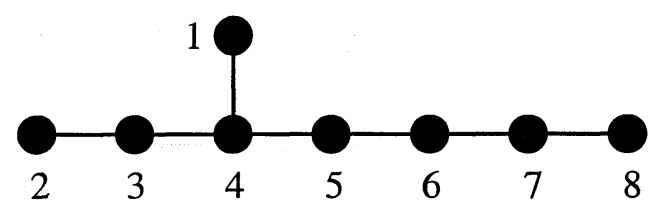

Figure 1: Numbering of simple roots of $E_{8(8)}$

\footnotetext{
${ }^{3}$ Modulo the subtlety that the two singleton irreps of $S L(2, \mathbb{R})$ have the same eigenvalue of the Casimir.
} 
They are

$$
\begin{array}{ll}
e_{1}=12 G^{4567}, & f_{1}=12 G^{1238} \\
e_{2}=G^{1}{ }_{2}, & f_{2}=G^{2}{ }_{1}, \\
e_{3}=G^{2}{ }_{3}, & f_{3}=G^{3}{ }_{2}, \\
e_{4}=G^{3}{ }_{4}, & f_{4}=G^{4}{ }_{3}, \\
e_{5}=G^{4}{ }_{5}, & f_{5}=G^{5}{ }_{4}, \\
e_{6}=G^{5}{ }_{6}, & f_{6}=G^{6}{ }_{5}, \\
e_{7}=G^{6}{ }_{7}, & f_{7}=G^{7}{ }_{6}, \\
e_{8}=E^{78}, & f_{8}=F_{78} .
\end{array}
$$

The generators of the Cartan subalgebra are given by

$$
\begin{aligned}
h_{1} & =G^{4}{ }_{4}+G^{5}{ }_{5}+G^{6}{ }_{6}+G^{7}{ }_{7}=-G^{1}{ }_{1}-G^{2}{ }_{2}-G^{3}{ }_{3}-G^{8}{ }_{8}, \\
h_{2} & =G^{1}{ }_{1}-G^{2}{ }_{2}, \\
h_{3} & =G^{2}{ }_{2}-G^{3}{ }_{3} \\
h_{4} & =G^{3}{ }_{3}-G^{4}{ }_{4}, \\
h_{5} & =G^{4}{ }_{4}-G^{5}{ }_{5}, \\
h_{6} & =G^{5}{ }_{5}-G^{6}{ }_{6}, \\
h_{7} & =G^{6}{ }_{6}-G^{7}{ }_{7}, \\
h_{8} & =G^{7}{ }_{7}+G^{8}{ }_{8}-\frac{1}{2} H .
\end{aligned}
$$

Comparison with the formulas given in the appendix of [26] shows that the basis of coordinate vs. momentum variables used there differs from ours by the choice of polarization (or "Fourier transformation"). Accordingly, the linearly realized subgroup exposed there is also different from ours.

\section{Oscillator Representation}

In the coordinate representation, the linearly realized $S L(8, \mathbb{R})$ subalgebra of $E_{7(7)}$ plays a distinguished role. As is well known, however, there is another basis of $E_{7(7)}$, which is equally important in supergravity and superstring theory, where $S L(8, \mathbb{R})$ is replaced by $S U(8)$ [10]. In this section, we demonstrate that the full $E_{8(8)}$ Lie algebra can be rewritten in terms of this complex basis. The change of basis is equivalent to the replacement of coordinates and momenta by creation and annihilation operators, and thus to the replacement of the coordinate representation by a holomorphic 
(Bargmann-Fock) representation. This will allow us to establish the connection with the oscillator realization of $E_{7(7)}$ discovered already some time ago [19]. For this purpose, we introduce the creation and annihilation (or raising and lowering) operators

$$
\begin{aligned}
& a^{A B}:=\frac{1}{4 \sqrt{2}} \Gamma_{A B}^{i j}\left(X^{i j}-\mathrm{i} P_{i j}\right) \\
& a_{A B}:=\frac{1}{4 \sqrt{2}} \Gamma_{A B}^{i j}\left(X^{i j}+\mathrm{i} P_{i j}\right) \equiv\left(a^{A B}\right)^{\dagger}
\end{aligned}
$$

The normalization has been chosen such that

$$
\left[a_{A B}, a^{C D}\right]=\delta_{A B}^{C D}
$$

Substituting these operators into (7) and defining

$$
\begin{aligned}
G_{B}^{A} & :=-\frac{1}{4} \mathrm{i} G_{i j}^{-} \Gamma_{A B}^{i j}+\frac{1}{8} G_{i j k l}^{-} \Gamma_{A B}^{i j k l}=G_{B}{ }^{A}, \\
G^{A B C D} & :=\left(\frac{1}{48} \mathrm{i} G_{i k}^{+} \delta_{l j}-\frac{1}{16} G_{i j k l}^{+}\right) \Gamma_{[A B}^{i j} \Gamma_{C D]}^{k l},
\end{aligned}
$$

we obtain the singleton representation of $E_{7(7)}$ in the $S U(8)$ basis of [19]

$$
\begin{aligned}
G_{B}^{A}{ }_{B} & :=2 a^{A C} a_{B C}-\frac{1}{4} \delta_{B}^{A} a^{C D} a_{C D} \\
G^{A B C D} & :=\frac{1}{2} a^{[A B} a^{C D]}-\frac{1}{48} \epsilon^{A B C D E F G H} a_{E F} a_{G H}
\end{aligned}
$$

where $G^{A}{ }_{B}$ now generates the $S U(8)$ subgroup of $E_{7(7)}$. In deriving this result, we made use of the formulas (see e.g. the appendices of $[10,12]$ )

$$
\Gamma_{A B}^{i j k l}=-\frac{1}{24} \epsilon^{i j k l m n p q} \Gamma_{A B}^{m n p q}
$$

and

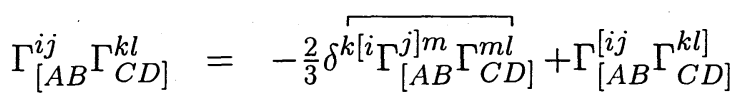

$$
\begin{aligned}
& \Gamma_{[A B}^{i k} \Gamma_{C D]}^{k j}=\frac{1}{24} \epsilon_{A B C D E F G H} \Gamma_{E F}^{i k} \Gamma_{G H}^{k j} \\
& \Gamma_{[A B}^{[i j} \Gamma_{C D]}^{k l]}=-\frac{1}{24} \epsilon_{A B C D E F G H} \Gamma_{E F}^{[i j} \Gamma_{G H}^{k l]} \\
& =+\frac{1}{24} \epsilon^{i j k l m n p q} \Gamma_{[A B}^{m n} \Gamma_{C D]}^{p q}
\end{aligned}
$$

We note the complex (anti)self-duality relation

$$
G_{A B C D} \equiv\left(G^{A B C D}\right)^{\dagger}=-\frac{1}{24} \epsilon_{A B C D E F G H} G^{E F G H}
$$

and the commutation relations

$$
\begin{aligned}
{\left[G_{B}^{A}, G^{C}{ }_{D}\right] } & =\delta_{B}^{C} G^{A}{ }_{D}-\delta_{D}^{A} G_{B}^{C} \\
{\left[G_{B}^{A}, G^{C D E F}\right] } & =-4 \delta_{B}^{[C} G^{D E F] A}-\frac{1}{2} \delta_{B}^{A} G^{C D E F}, \\
{\left[G^{A B C D}, G^{E F G H}\right] } & =\frac{1}{36} \epsilon^{A B C D I[E F G} G^{H]}{ }_{I} .
\end{aligned}
$$


In order to render the remaining $E_{8(8)}$ generators $S U(8)$ covariant, we define

$$
\begin{aligned}
& E^{A B}:=\frac{1}{4 \sqrt{2}} \Gamma_{A B}^{i j}\left(E^{i j}-\mathrm{i} E_{i j}\right)=y a^{A B}, \\
& E_{A B}:=\frac{1}{4 \sqrt{2}} \Gamma_{A B}^{i j}\left(E^{i j}+\mathrm{i} E_{i j}\right)=y a_{A B},
\end{aligned}
$$

The computation is more tedious for the generators which are cubic and quartic in the oscillators, and most conveniently done by checking the $E_{8(8)}$ algebra again. We have

$$
F=\frac{1}{2} p^{2}+2 y^{-2} I_{4}\left(a, a^{\dagger}\right)
$$

with the $S U(8)$ invariant expression for $I_{4}$ in terms of oscillators

$$
\begin{aligned}
I_{4}\left(a, a^{\dagger}\right) \equiv & I_{4}(X, P) \\
= & +\frac{1}{2}\left(a^{A B} a_{B C} a^{C D} a_{D A}+a_{A B} a^{B C} a_{C D} a^{D A}\right) \\
& -\frac{1}{8}\left(a^{A B} a_{A B} a^{C D} a_{C D}+a_{A B} a^{A B} a_{C D} a^{C D}\right) \\
& +\frac{1}{96} \epsilon^{A B C D E F G H} a_{A B} a_{C D} a_{E F} a_{G H} \\
& +\frac{1}{96} \epsilon_{A B C D E F G H} a^{A B} a^{C D} a^{E F} a^{G H}+\frac{547}{16} .
\end{aligned}
$$

Note that the normal-ordered version of $I_{4}$ (with all the annihilators to the right) is not $E_{7(7)}$ invariant, because

$$
I_{4}\left(a, a^{\dagger}\right)=: I_{4}\left(a, a^{\dagger}\right):-\frac{\mathrm{i}}{4} \mathcal{N}-49
$$

with the number operator

$$
\mathcal{N}:=a^{A B} a_{A B}
$$

which does not commute with $G^{A B C D}$.

The remaining generators are now straightforwardly deduced by commuting $F$ with $E^{A B}$ and $E_{A B}$ :

$$
\begin{aligned}
F^{A B}:= & \frac{1}{4 \sqrt{2}} \Gamma_{A B}^{i j}\left(F^{i j}-\mathrm{i} F_{i j}\right) \equiv \mathrm{i}\left[E^{A B}, F\right] \\
= & -p a^{A B}+\frac{\mathrm{i}}{2} y^{-1}\left(a^{A B} a_{C D} a^{C D}+a^{C D} a_{C D} a^{A B}\right) \\
& +4 \mathrm{i} y^{-1} a^{A C} a_{C D} a^{D B}-\frac{\mathrm{i}}{12} y^{-1} \epsilon^{A B C D E F G H} a_{C D} a_{E F} a_{G H} \\
F_{A B}:= & \frac{1}{4 \sqrt{2}} \Gamma_{A B}^{i j}\left(F^{i j}+\mathrm{i} F_{i j}\right) \equiv \mathrm{i}\left[E_{A B}, F\right] \\
= & -p a_{A B}-\frac{\mathrm{i}}{2} y^{-1}\left(a_{A B} a^{C D} a_{C D}+a_{C D} a^{C D} a_{A B}\right) \\
& -4 \mathrm{i} y^{-1} a_{A C} a^{C D} a_{D B}+\frac{\mathrm{i}}{12} y^{-1} \epsilon_{A B C D E F G H} a^{C D} a^{E F} a^{G H}
\end{aligned}
$$


These generators now transform covariantly under the $S U(8)$ group

$$
\begin{aligned}
& {\left[G_{B}^{A}, E_{C D}\right]=\delta_{C}^{A} E_{D B}-\delta_{D}^{A} E_{C B}+\frac{1}{4} \delta_{B}^{A} E_{C D},} \\
& {\left[G_{B}^{A}, F_{C D}\right]=\delta_{C}^{A} F_{D B}-\delta_{D}^{A} F_{C B}+\frac{1}{4} \delta_{B}^{A} F_{C D},}
\end{aligned}
$$

and the remaining part of $E_{7(7)}$ acts as

$$
\begin{aligned}
& {\left[G^{A B C D}, E_{E F}\right]=-\delta_{E F}^{[A B} E^{C D]},} \\
& {\left[G^{A B C D}, F_{E F}\right]=-\delta_{E F}^{[A B} F^{C D]} .}
\end{aligned}
$$

Furthermore,

$$
\begin{aligned}
& {\left[E_{A B}, E^{C D}\right]=2 \delta_{A B}^{C D} E} \\
& {\left[F_{A B}, F^{C D}\right]=2 \delta_{A B}^{C D} F} \\
& {\left[E^{A B}, F^{C D}\right]=-12 \mathrm{i} G^{A B C D},} \\
& {\left[E_{A B}, F^{C D}\right]=-2 \mathrm{i} \delta_{[A}^{[C} G^{D]}{ }_{B]}-\delta_{A B}^{C D} H .}
\end{aligned}
$$

It remains to discuss the $S L(2, \mathbb{R})$ subgroup, for which we likewise switch to a complex basis (the $S U(1,1)$ basis)

$$
\begin{aligned}
L_{0} & :=\frac{1}{2}(E+F)=\frac{1}{4}\left(p^{2}+y^{2}\right)+y^{-2} I_{4}(X, P), \\
L_{1} & :=\frac{1}{2}(E-F+\mathrm{i} H)=\frac{1}{4}(y+\mathrm{i} p)^{2}-y^{-2} I_{4}(X, P), \\
L_{-1} & :=\frac{1}{2}(E-F-\mathrm{i} H)=\frac{1}{4}(y-\mathrm{i} p)^{2}-y^{-2} I_{4}(X, P),
\end{aligned}
$$

In the absence of the term containing $y^{-2}$, it would again be convenient to employ creation and annihilation operators $b:=\frac{1}{\sqrt{2}}(y+i p)$ and $b^{\dagger}=\frac{1}{\sqrt{2}}(y-$ $i p)$ to recover the well known singleton representation of $S U(1,1)$, but for non-vanishing value of the quartic $E_{7(7)}$ invariant it is not possible to switch this term off. The presence of $y^{-1}$ and $y^{-2}$ makes it somewhat awkward to express all generators in terms of creation and annihilation operators, so one might prefer to keep the coordinate representation in this sector. The commutation relations are, however, not affected by the choice of variables.

$$
\left[L_{0}, L_{ \pm 1}\right]=\mp L_{ \pm 1}, \quad\left[L_{+1}, L_{-1}\right]=2 L_{0} .
$$

This basis is no longer hermitean, but

$$
L_{0}^{\dagger}=L_{0}, \quad\left(L_{ \pm 1}\right)^{\dagger}=L_{\mp 1}
$$

Diagonalizing the new hermitean generator $2 L_{0}$ instead of $H$ we obtain an alternative 5 -graded decomposition

$$
\mathfrak{e}_{8}=\mathfrak{k}^{-2} \oplus \mathfrak{k}^{-1} \oplus \mathfrak{k}^{0} \oplus \mathfrak{k}^{+1} \oplus \mathfrak{k}^{+2} .
$$


such that

$$
\left(\mathfrak{k}^{0}\right)^{\dagger}=\mathfrak{k}^{0}, \quad\left(\mathfrak{k}^{ \pm 1}\right)^{\dagger}=\mathfrak{k}^{\mp 1}, \quad\left(\mathfrak{k}^{ \pm 2}\right)^{\dagger}=\mathfrak{k}^{\mp 2}
$$

We note that, strictly speaking, the elements of $\mathfrak{k}^{n}$ by themselves do not belong to the real Lie algebra $\mathfrak{e}_{8}$ as defined in section 2 , but to its complexification. It is only the hermitean linear combinations which do.

The subspaces of grade \pm 2 are one-dimensional with generators $L_{\mp 1}$, the grade 0 space is spanned by the $E_{7(7)}$ generators (7) together with $L_{0}$ and the subspaces of grade \pm 1 are generated by $\mathcal{E}^{A B}, \mathcal{E}_{A B}$ and $\mathcal{F}^{A B}, \mathcal{F}_{A B}$ defined by

$$
\begin{aligned}
\mathcal{E}^{A B} & :=\frac{1}{\sqrt{2}}\left(E^{A B}+\mathrm{i} F^{A B}\right) \\
\mathcal{E}_{A B} & :=\frac{1}{\sqrt{2}}\left(E_{A B}+\mathrm{i} F_{A B}\right), \\
\mathcal{F}^{A B} & :=\frac{1}{\sqrt{2}}\left(E^{A B}-\mathrm{i} F^{A B}\right), \\
\mathcal{F}_{A B} & :=\frac{1}{\sqrt{2}}\left(E_{A B}-\mathrm{i} F_{A B}\right),
\end{aligned}
$$

respectively. In terms of these generators all structure constants are real:

$$
\begin{aligned}
{\left[L_{+1}, \mathcal{E}^{A B}\right] } & =2 \mathcal{F}^{A B} \\
{\left[L_{-1}, \mathcal{F}^{A B}\right] } & =-2 \mathcal{E}^{A B} \\
{\left[\mathcal{E}_{A B}, \mathcal{E}^{C D}\right] } & =2 L_{-1} \\
{\left[\mathcal{F}_{A B}, \mathcal{F}^{C D}\right] } & =2 L_{+1} \\
{\left[\mathcal{E}^{A B}, \mathcal{F}^{C D}\right] } & =-12 G^{A B C D} \\
{\left[\mathcal{E}_{A B}, \mathcal{F}^{C D}\right] } & =-2 \delta_{[A}^{[C} G^{D]}{ }_{B]}+2 \delta_{A B}^{C D} L_{0}
\end{aligned}
$$

\section{Decompositions and Truncations}

\section{1 $E_{7(7)} \times S L(2, \mathbb{R})$ decomposition of the minimal unitary representation of $E_{8(8)}$}

A non-compact group $G$ admits unitary representations of the lowest weight (or highest weight) type if and only if the quotient $G / K$ of $G$ with respect to its maximal compact subgroup $K$ is an Hermitean symmetric space [20, 21]. From this theorem it follows that the simple non-compact groups that admit lowest (highest) weight unitary representations are $S O(n, 2), S U(n, m)$, $S O^{*}(2 n), S p(2 n, \mathbb{R}), E_{6(-14)}$, and $E_{7(-24)}$. The unitary lowest (highest) weight representations belong to the holomorphic (anti-holomorphic) discrete series and within these representations the spectrum of, at least, one 
generator is bounded from below (above). More generally, a non-compact group $G$ admits representations belonging to the discrete series if it has the same rank as its maximal compact subgroup ${ }^{4}$. Thus the non-compact group $E_{8(8)}$ as well as $E_{7(7)}$ admit discrete series representations. However, they are not of the lowest or highest weight type.

In this subsection we will analyze the decomposition of the minimal representation of $E_{8(8)}$ with respect to its subgroup $E_{7(7)} \times S L(2, \mathbb{R})$, but using the complex basis of the last section. The group $S U(1,1)$ admits holomorphic and anti-holomorphic unitary representations, and they exhaust the list of discrete series representations for $S U(1,1)$. (This is not true for higher rank non-compact groups admitting such representations.) As mentioned earlier, the realization of the $S U(1,1)$ subgroup within the minimal unitary representation of $E_{8(8)}$ is precisely of the form that arises in conformal quantum mechanics [11]. This is perhaps not surprising since we obtained our realization from the geometric action of $E_{8(8)}$ as a quasi-conformal group in 57 dimensions [18].

By comparison of the $S U(1,1)$ subgroup (45) with that of [11] it follows that the coupling constant $g$ in conformal quantum mechanics is simply

$$
g=4 I_{4}(X, P)
$$

in our realization. The quadratic Casimir of $S U(1,1)$ is

$$
\mathcal{C}_{2}[S U(1,1)]=L_{0}^{2}-\frac{1}{2}\left(L_{1} L_{-1}+L_{-1} L_{1}\right)=I_{4}(X, P)-\frac{3}{16} .
$$

Thus for a given eigenvalue of $I_{4}(X, P)$, we are led to a definite unitary realization of $S U(1,1)$. As we showed above, $I_{4}(X, P)$ is simply the quadratic Casimir operator of $E_{7(7)}$ (cf. (15)) that commutes with $S U(1,1)$, up to an additive normal ordering constant. Hence classifying all the possible eigenvalues of $I_{4}(X, P)$ within the minimal unitary realization of $E_{8(8)}$ is equivalent to giving the decomposition of the $E_{8(8)}$ representation with respect to $E_{7(7)} \times S U(1,1)^{5}$. Unitarity requires the eigenvalues of the Casimir operators to be real. Therefore all the eigenvalues of $I_{4}$ must be real. As we showed above, the realization of $E_{7(7)}$ within $E_{8(8)}$ coincides with the singletonic oscillator realization of $E_{7(7)}$ [19]. The oscillator realization of $E_{7(7)}$ leads to an infinitely reducible unitary representation [19]. Hence the minimal representation of $E_{8(8)}$ will be infinitely reducible with respect to $E_{7(7)} \times S U(1,1)$.

\footnotetext{
${ }^{4}$ For an excellent introduction the general theory of unitary representations of noncompact groups see [28].

${ }^{5}$ Here we should note that in certain exceptional cases the eigenvalue of the quadratic Casimir operator may not uniquely label the unitary irreducible representation of $S U(1,1)$. In such cases one needs to use additional labels such as the eigenvalues of $L_{0}$.
} 
Denoting the eigenvalues of the quadratic Casimir operator $S U(1,1)$ as

$$
\mathcal{C}_{2}[S U(1,1)]=j(j-1)
$$

we find that

$$
j=\frac{1}{2} \pm \sqrt{I_{4}+\frac{1}{16}}
$$

Depending on the eigenvalue of $I_{4}$ we will be led to one of the well-known series of representations of $S U(1,1) \quad[2,28]$

(a) Continuous principal series:

$$
\begin{aligned}
j & =\frac{1}{2}-\mathrm{i} \rho, \quad 0<\rho<\infty, \\
j(j-1) & =-\left(\frac{1}{4}+\rho^{2}\right)<-\frac{1}{4}
\end{aligned}
$$

with the eigenvalues $\ell$ of $L_{0}$ unbounded from above and from below

$$
\ell=\ell_{0}, \ell_{0} \pm 1, \ell_{0} \pm 2, \ldots \quad\left(\ell_{0} \in \mathbb{R}\right)
$$

(b) Continuous supplementary series:

$$
\left|j-\frac{1}{2}\right|<\frac{1}{2}-\left|\ell_{0}\right|, \quad\left(j, \ell_{0} \in \mathbb{R}\right)
$$

again with unbounded eigenvalues $\ell$ of $L_{0}$ in both directions.

(c) Holomorphic discrete series $D^{+}(j)$ (lowest weight irreps):

$$
\begin{aligned}
& j>0, \quad \ell_{0}=j, \quad(j \in \mathbb{R}) \\
& \ell=j, j+1, j+2, \ldots
\end{aligned}
$$

(c) Discrete series $D^{-}(j)$ (highest weight irreps):

$$
\begin{aligned}
& j>0, \quad \ell_{0}=-j, \quad(j \in \mathbb{R}) \\
& \ell=-j,-j-1,-j-2, \ldots
\end{aligned}
$$

For vanishing quartic invariant $I_{4}$ we find

$$
j=\frac{1}{4} \text { or } j=\frac{3}{4}
$$

corresponding to the two singleton irreps of $S U(1,1)$. Note that the eigenvalues of the Casimir operator for the two singleton irreps coincide. They are distinguished by the value of $j$ which is the eigenvalue of $L_{0}$ on the corresponding lowest weight vector. 


\subsection{The $S U(2,1)$ truncation of $E_{8(8)}$}

To give the decomposition of the minimal representation of $E_{8(8)}$ w.r.t. its $E_{7(7)} \times S L(2, R)$ subgroup we need to determine all possible eigenvalues of $I_{4}(X, P)$ within our realization. Since this determination appears to be rather complicated, we shall study this question in a somewhat simpler setting by truncating $E_{8(8)}$ to a special subgroup. $E_{8(8)}$ has the subgroup $E_{6(2)} \times S U(2,1)$, where $E_{6(2)}$ has the maximal compact subgroup $S U(6) \times S U(2)$. It is instructive to truncate our realization to the $S U(2,1)$ subgroup, which is one of the minimal subgroups that admit a non-trivial 5 -grading. This is achieved by introducing coordinate $x$ and momentum $p_{x}$ corresponding to the symplectic trace components of $X^{i j}$ and $P_{i j}$, respectively:

$$
\begin{aligned}
x & :=-\frac{\sqrt{2}}{4} \Omega_{i j} X^{i j}, \\
p_{x} & :=\frac{\sqrt{2}}{4} \Omega^{i j} P_{i j},
\end{aligned}
$$

and throwing away the symplectic traceless components in our realization. The matrix $\Omega_{i j}$ is the symplectic metric defined by

$$
\Omega_{i j}:=\left(\begin{array}{cc}
0 & -\mathbb{1} \\
\mathbb{1} & 0
\end{array}\right)
$$

and $\Omega^{i j}$ is its inverse: $\Omega_{i j} \Omega^{j k}=\delta_{i}^{k}$. The generators $x$ and $p_{x}$ obey the canonical commutation relation

$$
\left[x, p_{x}\right]=\mathrm{i} .
$$

The resulting expressions for the generators of $S U(2,1)$ are

$$
\begin{aligned}
E & :=\frac{1}{2} y^{2}, \\
E_{\uparrow} & :=y x, \\
E_{\downarrow} & :=y p_{x}, \\
H & :=\frac{1}{2}\left(y p_{y}+p_{y} y\right) \\
A & :=-\frac{3}{4}\left(x^{2}+p_{x}^{2}\right) \\
F_{\uparrow} & :=-x p_{y}-\frac{1}{2} y^{-1}\left(x p_{x} x+p_{x}^{3}\right), \\
F_{\downarrow} & :=p_{x} p_{y}-\frac{1}{2} y^{-1}\left(p_{x} x p_{x}+x^{3}\right), \\
F & :=\frac{1}{2} p_{y}^{2}+\frac{1}{8} y^{-2}\left(x^{2}+p_{x}^{2}\right)^{2}-\frac{1}{8} y^{-2},
\end{aligned}
$$


The 5-grading in the above basis is with respect to $H$ which is the noncompact dilatation generator. To understand the resulting representation of $S U(2,1)$ it is useful to go to the basis in which the Lie algebra of $S U(2,1)$ has a 3 -grading w.r.t. the compact $U(1)$ generator that commutes with the $S U(2)$ subgroup.

$$
S U(2,1)=\left(\begin{array}{c}
L_{-} \\
K_{-}
\end{array}\right) \oplus\left(\begin{array}{c}
J_{i} \\
J_{0}
\end{array}\right) \oplus\left(\begin{array}{c}
L_{+} \\
K_{+}
\end{array}\right)
$$

The compact $U(1)$ generator is

$$
J_{0}=\frac{1}{2}\left(E+F-\frac{2}{3} A\right)=L_{0}-\frac{1}{3} A
$$

and the $S U(2)$ generators are

$$
\begin{aligned}
J_{1} & =\frac{1}{2 \sqrt{2}}\left(E_{\uparrow}+F_{\downarrow}\right), \\
J_{2} & =\frac{1}{2 \sqrt{2}}\left(E_{\downarrow}+F_{\uparrow}\right), \\
J_{3} & =\frac{1}{4}(E+F+2 A),
\end{aligned}
$$

with the commutation relations

$$
\begin{aligned}
& {\left[J_{i}, J_{j}\right]=\mathrm{i} \epsilon_{i j k} J_{k}, \quad(i, j, k=1,2,3)} \\
& {\left[J_{0}, J_{i}\right]=0 .}
\end{aligned}
$$

The grade \pm 1 generators are

$$
\begin{aligned}
K_{ \pm} & =\left(E_{\uparrow}-F_{\downarrow}\right) \mp \mathrm{i}\left(E_{\downarrow}-F_{\uparrow}\right), \\
L_{ \pm} & =\frac{1}{2}(E-F \mp \mathrm{i} H),
\end{aligned}
$$

satisfying

$$
\begin{aligned}
& {\left[J_{0}, K_{ \pm}\right]= \pm K_{ \pm},} \\
& {\left[J_{0}, L_{ \pm}\right]= \pm L_{ \pm}}
\end{aligned}
$$

More explicitly we have

$$
\begin{aligned}
K_{ \pm} & =\left\{\left(y \mp i p_{y}\right)+\frac{1}{2} y^{-1}\left[1+\left(x^{2}+p_{x}^{2}\right)\right]\right\}\left(x \mp i p_{x}\right) \\
L_{ \pm} & =\left\{\frac{1}{4}\left(y \mp i p_{y}\right)^{2}-\frac{1}{16} y^{-2}\left[-1+\left(x^{2}+p_{x}^{2}\right)^{2}\right]\right\}
\end{aligned}
$$

The generator $J_{0}$ that determines the 3 -grading of $S U(2,1)$ w.r.t. maximal compact subgroup $S U(2) \times U(1)$ is manifestly positive definite. In terms of the annihilation and creation operators $a_{x}, a_{x}^{\dagger}$ the generator $J_{0}$ takes the form:

$$
J_{0}=\frac{1}{4}\left\{y^{2}+p_{y}^{2}+y^{-2} N_{x}\left(N_{x}+1\right)+2 N_{x}+1\right\}
$$


where $N_{x}=a_{x}^{\dagger} a_{x}$ is the number operator. This implies that the resulting unitary irreducible representations of $S U(2,1)$ must be of the lowest weight type (positive energy). Now the quadratic Casimir operator of $S U(2,1)$ is given by

$$
\begin{aligned}
\mathcal{C}_{2}[S U(2,1)]= & \frac{1}{2}(E F+F E)-\frac{1}{4} H^{2}+\frac{1}{3} A^{2} \\
& +\frac{1}{4}\left(E_{\uparrow} F_{\downarrow}+F_{\downarrow} E_{\uparrow}\right)+\frac{1}{4}\left(E_{\downarrow} F_{\uparrow}+F_{\uparrow} E_{\downarrow}\right)
\end{aligned}
$$

Substituting the expressions for the generators we find that the the quadratic Casimir of $S U(2,1)$ becomes simply a $c$-number, namely

$$
\mathcal{C}_{2}[S U(2,1)]=-\frac{3}{16}
$$

which suggests that the representation may be irreducible. To have an irreducible representation the cubic Casimir must likewise reduce to a $c$-number. However, we can study the irreducibility of the representation without having to calculate the cubic Casimir. Since we know that our representation is of the lowest weight type we can use the fact that a unitary irrep of the lowest weight type is uniquely determined by a set of states $|\Omega\rangle$ transforming irreducibly under the maximal compact subgroup $S U(2) \times U(1)$ and that are annihilated by all the generators of grade -1 under the 3 -grading. Thus we need to find all such states that are annihilated by $K_{-}$and $L_{-}$:

$$
\begin{aligned}
& K_{-}|\Omega\rangle=2\left(a_{y}+\frac{1}{\sqrt{2}} y^{-1}\left(N_{x}+1\right)\right) a_{x}|\Omega\rangle=0 \\
& L_{-}|\Omega\rangle=\frac{1}{2}\left(a_{y}^{2}-\frac{1}{2} y^{-2} N_{x}\left(N_{x}+1\right)\right)|\Omega\rangle=0
\end{aligned}
$$

and that transform irreducibly under the maximal compact subgroup $S U(2) \times$ $U(1)$. The only normalizable state satisfying these conditions is the one particle excited state

$$
a_{y}^{\dagger}|0\rangle
$$

where the vacuum state $|0\rangle$ is annihilated by both annihilation operators

$$
\begin{aligned}
& a_{x}|0\rangle=0 \\
& a_{y}|0\rangle=0
\end{aligned}
$$

This state is a singlet of $S U(2)$ and has the $U(1)$ charge 1 . This proves that the minimal realization of $S U(2,1)$ obtained by truncation of the minimal representation of $E_{8(8)}$ is also irreducible.

The subgroup $E_{7(7)} \times S U(1,1)$ of $E_{8(8)}$ under truncation to $S U(2,1)$ reduces to $U(1) \times S U(1,1)$. The generators of $S U(1,1)$ subgroup of $S U(2,1)$ 
are given by

$$
\begin{aligned}
L_{+} & =\frac{1}{2}(E-F-i H) \\
& =\left\{\frac{1}{4}\left(y-i p_{y}\right)^{2}-\frac{1}{16} y^{-2}\left[-1+\left(x^{2}+p_{x}^{2}\right)^{2}\right]\right\} \\
L_{-} & =\frac{1}{2}(E-F+i H) \\
& =\left\{\frac{1}{4}\left(y+i p_{y}\right)^{2}-\frac{1}{16} y^{-2}\left[-1+\left(x^{2}+p_{x}^{2}\right)^{2}\right]\right\} \\
L_{0} & =\frac{1}{2}(E+F) \\
& =\left\{\frac{1}{4}\left(y^{2}+p_{y}^{2}\right)+\frac{1}{16} y^{-2}\left[-1+\left(x^{2}+p_{x}^{2}\right)^{2}\right]\right\}
\end{aligned}
$$

They are therefore the analogs of the generators(45). The generator of $U(1)$ that commutes with $S U(1,1)$ is simply $A$. In this truncation the quartic $E_{7(7)}$ invariant reduces to

$$
I_{4}=\frac{1}{16}\left[\left(x^{2}+p_{x}^{2}\right)^{2}-1\right]
$$

which can be written in terms of the annihilation and creation operators as

$$
I_{4}=\frac{1}{4}\left\{\left(a_{x}^{\dagger} a_{x}\right)\left(a_{x}^{\dagger} a_{x}+1\right)\right\}
$$

with the (obvious) identifications $a_{x}=-\frac{\sqrt{2}}{4} \Omega^{A B} a_{A B}$ and $a_{x}^{\dagger}=\frac{\sqrt{2}}{4} \Omega_{A B} a^{A B}$. Since the eigenvalues of the number operator $a^{\dagger} a$ are non-negative integers $\mathrm{n}$, the eigenvalues of $I_{4}$ are simply $\frac{1}{4} n(n+1)$. In fact the realization of $S U(1,1)$ in this case leads to unitary lowest weight representations of the type studied in [11]. For $n=0$ we get the singleton irreps of $S U(1,1)$ corresponding to the values $j=\frac{1}{4}$ and $\frac{3}{4}$. Thus the minimal unitary representation of $S U(2,1)$ decomposes into a discretely infinite set of irreps of $S U(1,1)$ labelled by the eigenvalues of the $U(1)$ generator which is the analog of the $E_{7(7)}$ subgroup of $E_{8(8)}$ for the $S U(2,1)$ truncation.

\section{Outlook}

The finite dimensional conformal group $S U(1,1)$ is well known to possess an infinite dimensional extension (the Witt-Virasoro group). One may therefore ask whether there exists a generalization of this fact to $E_{8(8)}$. In other words, does there exist an infinite dimensional Lie algebra (or Lie superalgebra) that contains the Witt-Virasoro algebra and $E_{8(8)}$ at the same time? While there appears to be no linear Lie algebra with this property (and no finite dimensional Lie superalgebra, either), an infinite dimensional non-linear algebra of $\mathcal{W}$-type does exist. It is a nonlinear quasi-superconformal algebra denoted as $Q E_{8(8)}$ [4]. The quasi-superconformal algebras in two dimensions were first introduced in[32] and further systematized in[3]. They were generalized 
in[33] where two infinite families of nonlinear quasi-superconformal algebras were introduced. A classification of complex forms of quasi-superconformal algebras was given in [15]. In [4] a complete classification and a unified realization of the real forms of quasi-superconformal algebras were given.

In the infinite central charge limit the exceptional quasi-superconformal algebra $Q E_{8(8)}$ has the Lie algebra $E_{8(8)}$ as a maximal finite dimensional simple Lie subalgebra. The realization of $Q E_{8(8)}$ given in[4] involves 56 dimension $1 / 2$ bosons and a dilaton, which leads to a realization of $E_{8(8)}$ in the infinite central charge limit. It will be important to understand if the resulting realization can be related to the one given here. Furthermore one would like to know if one could use the methods of [4] to give a unified realization of the unitary representations of non-compact groups that act as quasi-conformal groups as formulated in [18], thus generalizing our minimal realization $E_{8(8)}$ to all such noncompact groups.

Acknowledgments: We are grateful to B. Pioline and A. Waldron for discussions, and for sending us an advance copy of ref. [26]. We would also like to thank R. Brylinski for bringing the references [35] and [17] to our attention and for helpful discussions.

\section{References}

[1] J. Adams, J. Huang and D.A. Vogan. Functions on the Model Orbit in $E_{8}$ Representation Theory 2, 224 (1998).

[2] V. Bargmann. Irreducible unitary representations of the Lorentz group Ann. Math. 48, 568 (1947).

[3] M. Bershadsky. Conformal field theories via Hamiltonian reduction Comm. Math. Phys. 139, 67 (1993).

[4] B. Bina and M. Günaydin. Real forms of non-linear superconformal and quasi-superconformal algebras and their unified realization. Nucl. Phys. B502, 713 (1997).

[5] R. Brylinski, B. Kostant. Minimal representations of $e_{6}, e_{7}$, and $e_{8}$ and the generalized Capelli identity. Proc. Natl. Aca.d. Sci. 91, 2469 (1994)

[6] R. Brylinski, B. Kostant. Minimal representations, geometric quantization, and unitarity. Proc. Natl. Acad. Sci. 91, 6026 (1994)

[7] R. Brylinski, B. Kostant. Lagrangian models of minimal representations of $e_{6}, e_{7}$ and $e_{8}$. Prog. Math. 131, 13 (1995) 
[8] R. Brylinski, B. Kostant. Geometric quantization and holomorphic halfform models of unitary minimal representations I Preprint (1996)

[9] P. Claus, M. Derix, R. Kallosh, J. Kumar, P. Townsend, A. Van Proeyen. Black holes and superconformal mechanics. Phys. Rev. Lett. 81, 4553 (1998)

[10] E. Cremmer, B. Julia. The $S O(8)$ supergravity. Nucl. Phys. B159, 141 (1979)

[11] V. de Alfaro, S. Fubini, G. Furlan. Conformal invariance in quantum mechanics. Nuovo Cim. A34, 569 (1976)

[12] B. de Wit, H. Nicolai. $d=11$ supergravity with local $S U(8)$ invariance Nucl. Phys. B274, 363 (1986)

[13] S. Ferrara, M. Günaydin. Orbits of exceptional groups, duality and BPS states in string theory. Int. J. Mod. Phys., A13, 2075 (1998) [hep-th/9708025]

[14] S. Ferrara, J. Maldacena. Branes, central charges and $U$-duality invariant BPS conditions. Class. Quant. Grav., 15, 749 (1998) [hepth/9706097]

[15] E.S. Fradkin and V. Yu. Linetsky. Phys. Lett. B275, 345 (1992); ibid. B282, 352 ( 1992).

[16] G. W. Gibbons and P. K. Townsend. Black holes and Calogero models. Phys. Lett. B454, 187 (1999) [hep-th/9812034]

[17] B. Gross and N. Wallach. A distinguished family of unitary representations for the exceptional groups of real rank $=4$, in Lie Theory and Geometry: in Honor of B. Kostant, J.-L. Brylinski, R. Brylinski, V. Guillemin, V. Kac, eds, Progress in Mathematics 123, Birkhauser, Boston (1994), 289-304.

[18] M. Günaydin, K. Koepsell, H. Nicolai. Conformal and quasiconformal realizations of exceptional Lie groups (2000). Commun. Math. Phys. 221, 57 (2001) [hep-th/0008063]

[19] M. Günaydin, C. Saçlioğlu. Oscillator like unitary representations of noncompact groups with a Jordan structure and the noncompact groups of supergravity. Commun. Math. Phys. 87, 159 (1982)

[20] Harish-Chandra. Representations of semisimple Lie groups. V. Am. J. Math. 78, 1 (1956) 
[21] Harish-Chandra. Representations of semisimple Lie groups VI. Integrable and square- integrable representations. Am. J. Math. 78, 564 (1956)

[22] A. Joseph. Minimal realizations and spectrum generating algebras. Commun. Math. Phys. 36, 325 (1974)

[23] A. Joseph. The minimal orbit in a simple Lie algebra and its associated maximal ideal. Ann. Sci. Ec. Norm. Super., IV. Ser. 9, 1 (1976)

[24] R. Kallosh, B. Kol. E(7) symmetric area of black hole horizon Phys. Rev. D53, 5344 (1996)

[25] D. Kazhdan, G. Savin. The smallest representation of simply laced groups Israel Math. Conf. Proceedings, Piatetski-Shapiro Festschrift 2, 209 (1990)

[26] D. Kazhdan, B. Pioline, A. Waldron. Minimal representations, spherical vectors, and exceptional theta series I. Commun. Math. Phys. 226, 1 (2002) [hep-th/0107222]

[27] See: http://www.lpthe.jussieu.fr/ pioline/minrep/E8.h.

[28] A. Knapp. Representation theory of semisimple groups, an overview based on examples. Princeton Mathematical Series $\mathbf{3 6}$.

[29] K. Koepsell. PhD Thesis, Hamburg University (June 2001)

[30] J. Michelson, A. Strominger. Superconformal multi-black hole quantum mechanics. JHEP 09, 005 (1999)

[31] B. Pioline, H. Nicolai, J. Plefka, A. Waldron $R^{4}$ couplings, the fundamental membrane and theta correspondence JHEP 0103, 036 (2001)

[32] A. Polyakov. Int. J. Mod. Phys. A5, 833 (1990).

[33] L.J. Romans. Nucl. Phys. B357, 549 (1991).

[34] F. Sugino, P. Vanhove U-duality from matrix membrane partition function [hep-th/0107145]

[35] D. Vogan. Singular Unitary Representations in Non-commutative Harmonic Analysis and Lie Groups, J. Carmona and M. Vergne, eds. Springer Lecture Notes 880, Berlin, 1981, 506-535. 